electrons can not be neglected above the transition temperature $\left(T / T_{c} \gtrsim 1.1\right)$ in 'less dirty films'.

\author{
Ein einfacher Kohärenzindikator \\ von R. DÄNDLIKER und F. M. MottieR \\ (Brown Boveri Forschungszentrum, Baden)
} (1971).

Eine ausführliche Publikation ist erschienen in Z. angew. Math. Phys. 22, 369

\title{
Messungen orts- und zeitabhängiger Strömungen mit einer Laser-Dopplersonde
}

von P. D. Iten und J. Mastner

(Brown Boveri Forschungszentrum, Baden)

Je höher die räumliche Auflösung einer Dopplersonde, desto kürzer sind die durch die Streuteilchen erzeugten dopplerverschobenen Streulicht-Impulse. Ein solcher enthält typische 10-20 Perioden $T_{d}$ der Dopplerfrequenz $f_{d}=1 / T_{d}$. Mittels Spektrumanalysatoren kann $f_{d}$ somit nur auf $10-5 \%$ genau bestimmt werden. Durch direkte Messung der Nullstellen des Dopplersignals wird die Bestimmung der Dopplerfrequenz unabhängig von der spektralen Breite des Signals. Man erreicht dadurch eine Verbesserung der Genauigkeit um mindestens zwei Grössenordnungen. Mittels eines hybriden Signalverarbeitungssystems wird die Momentanfrequenz des Dopplersignals mit einer zeitlichen Auflösung von $\tau=4 T_{d}$ dargestellt. Es werden Strömungsprofile in einem quadratischen Kanal in der Umgebung einer parallel zur Strömungsrichtung gestellten Platte gezeigt. Bei schräg gestellter Platte wird der Utbergang von laminarer Strömung zur Kármánschen Wirbelstrasse und schliesslich zur Turbulenz beobachtet.

\section{Berechnung der spektralen und räumlichen Auflösung einer optischen Dopplersonde}

\author{
von B. Eliasson \\ (Brown Boveri Forschungszentrum, Baden)
}

In der optischen Dopplersonde wird ein Volumenelement einer strömenden Flüssigkeit oder eines Gases durch zwei kohärente "Gaussische» Lichtstrahlen, die sich kreuzen, beleuchtet. Das optische Streusignal von einem sich durch den Kreuzungspunkt bewegenden Teilchen wird einem der beiden Strahlen überlagert und die Schwebungsfrequenz in einem Photodetektor erzeugt. Der Frequenzverlauf und die Leistung des detektierten Signals sind von den Eigenschaften der "Gaussischen" Strahlen abhängig. Durch eine geeignete Fokussierung der beiden Strahlen können die räumliche und spektrale Auflösung des Systems optimiert werden. Es werden Kriterien für hohe spektrale und/oder hohe räumliche Auflösung und maximale Signalleistung angegeben. 